\title{
Correlação Feldstein-Horioka: indicador de mobilidade de capitais ou de solvência?
}

\author{
Feldstein-Horioka corelation: indicator \\ of capital mobility or solvency?
}

FABIANA ROCHA*,**

\begin{abstract}
RESUMO: O objetivo deste trabalho é utilizar os modelos macro abertos intertemporais, e os testes econométricos de solvência derivados deles, para analisar a correlação original de poupança-investimento de Feldstein e Horioka. A restrição de solvência implica que a poupança e o investimento são cointegrados com um coeficiente de um e, portanto, que a conta corrente é estacionária. Como a regressão da seção transversal de Feldstein-Horioka mede o coeficiente médio de longo prazo, é possível argumentar que ela está capturando o coeficiente unitário implícito na restrição de solvência e não alguma medida de mobilidade de capital.
\end{abstract}

PALAVRAS-CHAVE: Mobilidade de capitais; solvência; Feldstein-Horioka.

ABSTRACT: The purpose of this paper is to use the intertemporal open macro models, and the solvency econometric tests derived from them, to analyze the original Feldstein and Horioka saving-investment correlation. The solvency constraint implies that saving and investment are cointegrated with a coefficient of one and, therefore, that the current account is stationary. Since the Feldstein-Horioka cross-section regression measures the average longrun coefficient, it is possible then to argue that it is capturing the unit coefficient implied by the solvency constraint and not some measure of capital mobility.

KEYWORDS: Capital mobility; solvency; Feldstein-Horioka.

JEL Classification: C23; F31.

\section{INTRODUÇÃO}

Feldstein e Horioka (1980) sugeriram como medida do grau de mobilidade de capitais a correlação entre a poupança e o investimento. Com base numa amostra

\footnotetext{
* Departamento de Economia, Universidade de São Paulo, São Paulo/SP, Brasil. E-mail: frocha@usp.br. Submetido: 5/Janeiro/2002;Aprovado: 4/Abril/2002.

** Eu gostaria de agradecer ao professor Luiz Carlos Bresser-Pereira pelo incentivo e pelos comentários. Quaisquer erros são de minha inteira responsabilidade.
} 
de 16 países da OCDE obtiveram evidência de que poupança e investimento eram altamente correlacionados e concluíram que o grau de mobilidade de capitais em países industrializados era baixo, contrariando o senso comum de que esses países tinham poucas restrições ao movimento de capitais. Além disso, a correlação estimada era extremamente estável ao longo do tempo, a despeito da crença de que a mobilidade de capitais teria aumentado depois de meados dos anos 70. Murphy (1984), Obstfeld (1986), Dooley et al. (1987) e Wong (1990) também acharam evidência de uma associação alta entre poupança e investimento para países industrializados menores e para países em desenvolvimento, embora as correlações estimadas fossem na média mais baixas. Contudo, as correlações estimadas eram também menores no período anterior a meados dos anos 70 do que no período posterior. A regularidade dos resultados fez com que a correlação poupança-investimento de Feldstein e Horioka se tornasse um dos mais importantes "puzzles" em macroeconomia internacional (Obstfeld e Rogoff, 2000). ${ }^{1}$

O objetivo desse artigo é discutir o coeficiente de correlação entre poupança e investimento a partir de modelos de equilíbrio geral intertemporais. Sinn (1992) mencionou esses modelos para criticar as regressões de "cross-section" tradicionalmente usadas para testar a correlação poupança-investimento. Numa economia sem crescimento, os saldos em conta corrente quando somados devem ser iguais a zero, ou seja, déficits em conta corrente devem ser seguidos em algum momento por superávits em conta corrente de forma que a restrição orçamentária intertemporal seja atendida. Então, dado que o saldo em conta corrente é contabilmente igual à diferença entre poupança e investimento e que a média da poupança e do investimento ao longo do tempo devem ser aproximadamente iguais pela restrição orçamentária, regressões de "cross-section" criam um viés para cima na correlação investimento-poupança. O correto, então, seria estimar a correlação poupança-investimento para país individualmente com base em séries temporais. Coakley et al. (1996), por sua vez, usaram uma versão linear do modelo sugerido por Feldstein (1983) para mostrar que a condição de solvência requer que o saldo do balanço de pagamentos seja uma variável estacionária e, portanto, poupança e investimento cointegram com um coeficiente de longo prazo igual a 1 .

Utiliza-se aqui explicitamente um modelo intertemporal para chegar à mesma conclusão de Coakley et al. (1996). Dado que as regressões de "cross-section" somente podem ser vistas como relações de longo prazo $^{2}$, acredita-se que esta estrutura teórica é a referência correta para avaliar (ou reavaliar) a formulação original de Feldstein e Horioka. Na verdade, existe uma extensa literatura não relacionada que avalia econometricamente a condição de solvência ou sustentabilidade externa de diferentes economias (Husted [1992], Sawada [1994], Ahmed e Rogers [1995],

\footnotetext{
${ }^{1}$ Para críticas à interpretação da correlação poupança-investimento como indicador de mobilidade de capitais ver, entre outros, Obstfeld (1994).

${ }^{2}$ As regressões não podem ser vistas como relações estruturais (relações comportamentais num modelo teórico) nem como relações na forma reduzida (solução de um sistema).
} 
entre outros). Recorre-se a esta literatura para reler a equação original de Feldstein e Horioka. Com base nessa releitura é que se argumenta que a associação entre poupança e investimento não é um indicador de mobilidade de capitais mas uma medida de solvência e, portanto, não existe qualquer "puzzle".

$\mathrm{O}$ artigo está organizado da seguinte maneira. A segunda seção apresenta a equação original de Feldstein e Horioka e discute as diferentes especificações econométricas que têm sido utilizadas para estimá-la. A terceira seção mostra que os testes de solvência/sustentabilidade derivados de modelos intertemporais são equivalentes ao teste de mobilidade de capitais dado pela correlação poupança-investimento. A seção 4 resume as principais conclusões.

\section{A EQUAÇÃO DE FELDSTEIN-HORIOKA E SUAS DIFERENTES ESPECIFICAÇÕES ECONOMÉTRICAS}

Feldstein e Horioka (1980) propuseram avaliar o grau de mobilidade de capitais através da correlação entre poupança e investimento. Eles estimaram a seguinte regressão de "cross-section" :

$$
(I / Y)_{i}=a+b(S / Y)_{i}+u_{i}
$$

onde $(\mathrm{I} / \mathrm{Y})$ é a razão entre o investimento doméstico bruto e o produto nacional e $(S / Y)$ é a razão entre a poupança doméstica bruta e o produto nacional. i é um índice de país, a e b representam parâmetros a serem estimados e u é o termo de erro. Para países pequenos, b deveria ser próximo de zero sob a hipótese de perfeita mobilidade de capitais. Quando b é igual a zero não existe relação entre poupança doméstica e investimento doméstico. Por outro lado, se b é grande, o capital deve ser bastante móvel. Se b é igual a 1, por exemplo, então toda a poupança adicional é usada para financiar investimento doméstico. Originalmente as regressões baseadas na equação (1) usaram médias de longo prazo das razões poupança e investimento para um conjunto de países. O argumento era de que, dado que I/Y e $\mathrm{S} / \mathrm{Y}$ são procíclicos, o uso de dados anuais implicaria um viés para cima no coeficiente b. Dados médios, então, eliminariam os efeitos do ciclo econômico (ver, entre outros, Bayoumi (1990)).

Usando uma amostra de países da OCDE Feldstein e Horioka (1980) obtiveram uma estimativa para $\mathrm{b}$ de 0,89 , o que foi interpretado como indicação de que havia um baixo grau de mobilidade de capitais entre os países industrializados.

A equação (1) também foi estimada usando séries de tempo. $O$ uso de séries de tempo ao invés de "cross-sections" traria duas vantagens. Primeiro, evitaria o problema de viés de seleção de amostra típico dos estudos de "cross-section" (Wong, 1990) e evitaria o viés contra mobilidade de capitais resultante da correlação entre poupança e investimento introduzida pelos dados médios num contexto intertemporal (Sinn, 1992).

Frankel (1986,1991), Tesar (1991) e Sinn (1992) usaram variáveis em níveis: 


$$
(I / Y)_{t}=a+b(S / Y)_{t}+u_{t}
$$

Feldstein (1983), Feldstein and Bachetta (1991) e Bayoumi (1990) estimaram a equação em primeiras diferenças:

$$
\Delta \Delta(I / Y)_{t}=a+b \Delta(S / Y)_{t}+u_{t}
$$

Bayoumi (1990) trabalha com as primeiras diferenças para tornar as séries estacionárias. Contudo, a menos que exista uma relação de longo prazo entre poupança e investimento, a equação em diferenças está mal especificada (ela é sobreidentificada).

Mais recentemente, as regressões entre poupança e investimento usando séries de tempo começaram a ser estimadas usando-se técnicas de raiz unitária e cointegração.

Com dados temporais é necessário inicialmente verificar se I/Y e S/Y são não estacionários. Se este é o caso, ou seja, I/Y e S/Y são I(1), o teste de Feldstein e Horioka implica que $S / Y$ e I/Y são cointegrados com vetor de cointegração $(1,-1)$ ', dado que a hipótese nula de perfeita correlação entre poupança e investimento é Ho $(a, b)=(0,1)$ (Gundlach e Sinn, 1992). Para os países desenvolvidos testes de cointegração foram feitos em Miller (1988), Leachman (1991), De Haan e Siermann (1994), e Argimón e Roldán (1994). Para países em desenvolvimento não se tem conhecimento de testes desse tipo. Além disso, a hipótese de que S/Y e I/Y são cointegrados com vetor de cointegração $(1,-1)$ ' implica que a combinação linear S/Y-I/Y é I $(0)$. Dado que por definição a conta corrente é igual à diferença entre poupança e investimento, se não é possível rejeitar a hipótese de não estacionariedade da conta corrente pode-se concluir que existe mobilidade de capitais. ${ }^{3}$

\section{A ABORDAGEM DE RAIZ UNITÁRIA E COINTEGRAÇÃO DA CORRELAÇÃO POUPANÇA-INVESTIMENTO E OS TESTES DE SOLVÊNCIA}

Seguindo a vasta literatura sobre a possibilidade de déficits orçamentários permanentes do Governo ${ }^{4}$, alguns trabalhos que investigam a tendência de longo prazo da conta corrente apareceram. Nestes, solvência é entendida como a habilidade de um país gerar superávits suficientes no futuro para repagar a sua dívida existente, ou seja, o valor presente descontado dos saldos em conta corrente deve ser igual ao valor do estoque da dívida. Por outro lado, uma trajetória de déficits em conta corrente seria considerada sustentável quando a mudança esperada para

\footnotetext{
${ }^{3}$ Testes para estacionariedade da conta corrente de países em desenvolvimento foram feitos por Bagnai e Manzocchi (1996). Eles concluem que, em 14 dos 37 países examinados, parece haver um alto grau de mobilidade de capitais.

${ }^{4}$ Ver, por exemplo, Hamilton and Flavin (1986), Wilcox (1989) Trehan e Walsh (1991) e Hakkio e Rush (1991) para os Estados Unidos. Para o Brasil ver Pastore (1995), Rocha (1997), Issler e Lima (2000) e Luporini (2000).
} 
reverter a balança comercial para uma posição consistente com solvência ocorresse sem mudanças drásticas nas políticas correntes e/ou crise externa (Corsetti, Pesenti e Roubini (1998a, 1998b) e Milesi-Ferreti e Razin (1996a, 1996b).

Trehan e Walsh (1991) argumentam que se a mudança no estoque dos ativos domésticos mantidos por estrangeiros é estacionária, então os déficits externos são sustentáveis. Usando dados de 1947 a 1987 eles não são capazes de rejeitar não estacionariedade para a dívida americana. Husted (1992), por outro lado, argumenta que se exportações e importações (incluindo pagamentos de juros externos) são cointegrados então a restrição orçamentária intertemporal é atendida. Para o período 1960-1989 não é possível achar evidência de cointegração. Se uma quebra em 1983 é, contudo, considerada encontra-se evidência de cointegração. O teste de solvência de Ahmed e Rogers (1995) envolve cointegração entre exportações, importações e pagamentos líquidos de juros externos com vetor $(1,-1,-1)$. Para os Estados Unidos e Reino Unido eles concluem que a restrição de valor presente é atendida quando a amostra completa é considerada. Finalmente, Sawada (1994) testa solvência para 13 países altamente endividados. Para o Brasil, durante o período 19551990, há indicação de insolvência. Outros estudos para o Brasil são Ponta (1996) e Rocha e Bender (2000) que também concluem que as condições para o equilíbrio orçamentário intertemporal não são satisfeitas.

O objetivo dessa seção é usar os mesmos modelos intertemporais que deram origem aos testes de sustentabilidade/solvência e reinterpretá-los a partir dos testes da correlação investimento-poupança. A despeito da distinção entre sustentabilidade e solvência ressaltados anteriormente, os dois conceitos serão utilizados indistintamente no restante do artigo. ${ }^{5}$

Suponha uma economia aberta pequena com horizonte infinito que consome um único bem. $\mathrm{O}$ agente representativo maximiza:

$$
U_{t}=\lim _{t \rightarrow \infty}\left[u\left(C_{t}\right)+\beta u\left(C_{t+1}\right)+\beta^{2} u\left(C_{t+2}\right)+\ldots\right]=\sum_{s=t}^{\infty} \beta^{s-t} u(C s)
$$

Para simplificar assume-se que a taxa de juros mundial $\mathrm{r}$ é constante ao longo do tempo. O produto em cada período $Y$ é determinado pela função de produção $Y=A F(K)$, onde $F(K)$ tem as propriedades usuais. A economia começa no período t com estoque de capital $K_{t}$ e ativos externos líquidos $B_{t}$ acumulados no passado.

A identidade da conta corrente (de novo assumindo uma taxa de juros constante) estabelece que :

\footnotetext{
${ }^{5} \mathrm{O}$ mesmo procedimento é usado na literatura que discute desequilíbrios fiscais. Contudo, como argumentam Milesi-Ferreti e Razin (1996a,1996b) "the definition of sustainability based on solvency conditions is simple for fiscal imbalances, given that these can be associated (at least to some degree) with direct policy decisions on taxation and government expenditure. Defining sustainability is more complex in the case of current account imbalances, given that these reflect the interaction between savings and investment decisions of the government and domestic private agents, as well as the lending decisions of foreign investors. While government decisions can, to a first approximation, be taken as given, private sector decisions are going to depend on their perceptions regarding future government actions. Furthermore, a key relative price, the exchange rate, is a forward-looking variable that by definition depends on the future evolution of policy variables."
} 


$$
C A_{t}=B_{t+1}-B_{t}=Y_{t}+r B_{t}-C_{t}-G_{t}-I_{t}
$$

onde $I_{t}=K_{t+1}-K_{t}$. Rearranjando termos tem-se:

$$
(1+r) B_{t}=C_{t}+G_{t}+I_{t}-Y_{t}+B_{t+1}
$$

Fazendo interações sucessivas de (3.3) para a frente obtém-se :

$$
\sum_{s=t}^{\infty}\left(\frac{1}{1+r}\right)^{s-t}\left(C_{s}+I_{s}\right)+\left(\frac{1}{1+r}\right)^{T} B_{t+T+1}=(1+r) B_{t}+\sum_{s=t}^{\infty}\left(\frac{1}{1+r}\right)^{s-t}\left(Y_{s}-G_{s}\right)
$$

A fim de achar as condições necessárias para a maximização de $U t$ na equação (3.1) simplesmente substituem-se os níveis de consumo em (1) usando a identidade da conta corrente e obtém-se :

$$
U_{t}=\sum_{s=l}^{\infty} \beta^{s-t} u\left[(1+r) B_{s}-B_{s+1}+A_{s} F\left(\mathrm{~K}_{s}\right)-\left(K_{s+1}-K_{s}\right)-G_{s}\right]
$$

Maximizando com relação a $B_{s+1}$ e $K_{s+1}$ resulta em:

$$
\begin{aligned}
& u^{\prime}\left(C_{s}\right)=(1+r) \beta u^{\prime}\left(C_{s+1}\right), \\
& A_{s+1} F^{\prime}\left({ }_{s+1}^{K}\right)=r
\end{aligned}
$$

A condição (3.6) é a equação de Euler para o consumo e a condição (3.7) fornece a igualdade entre o produto marginal do capital e a taxa de juros mundial.

A condição de transversalidade

$$
\lim _{T \rightarrow \infty}\left(\frac{1}{1+r}\right)^{T} \mathrm{~B}_{t+T+1}=0
$$

deve sempre valer para um indivíduo maximizador. Ela é equivalente a:

$$
\begin{aligned}
& \sum_{s=t}^{\infty}\left(\frac{1}{1+r}\right)^{s-t}\left(C_{s}+I_{s}\right)=(1+r) B_{t}+\sum_{s=t}^{\infty}\left(\frac{1}{1+r}\right)^{s-t}\left(Y_{s}-G_{s}\right) \\
& \quad \text { ou } \\
& -B_{t}=\sum_{s=t}^{\infty}(1+r)^{-(s-t)-1}\left(Y_{s}-G_{s}-C_{s}-I_{s}\right)
\end{aligned}
$$

Se o termo no limite em (3.8) fosse negativo, a economia estaria consumindo e investindo mais do que o valor presente do seu produto por uma quantidade que nunca converge a zero. A economia está usando sistematicamente dívida nova para pagar dívida velha ao invés de transferir recursos reais aos seus credores através de $C+I<Y-G$. Tal esquema Ponzi, porém, não é possível indefinidamente. Se o termo no limite em (3.8) fosse por outro lado positivo, o valor presente dos recursos seria menor do que o valor presente do produto e os consumidores domésticos poderiam aumentar sua utilidade consumindo mais. Somente quando o termo no limite é exatamente igual a zero a economia está usando, assintoticamente, exatamente os recursos que sua restrição orçamentária permite.

Uma maneira útil de interpretar a equação (3.10) é fazer $Y_{s}-C_{s}-G_{s}=S_{s}$. Uma vez que numa economia com investimento $C A s=S s-I s$, a equação (3.10) se torna:

$$
-B_{t}=\sum_{s=t}^{\infty}(1+r)^{-(s-t)-1}\left(S_{s}-I_{s}\right)
$$


ou

$$
-B_{t}=\sum_{s=t}^{\infty}(1+r)^{-(s-t)-1}\left(C A_{s}\right)
$$

Se o país satisfaz sua restrição orçamentária intertemporal, o valor presente descontado dos saldos da conta corrente deve ser igual à quantidade necessária para repagar o principal e os juros sobre sua dívida inicial. Quando isso acontece tem-se solvência externa.

Se $\Delta S_{t}$ e $\Delta I_{t}$ são processos estacionários, pode-se demonstrar que a seguinte relação de cointegração implica sustentabilidade/solvência externa: ${ }^{6}$

$$
S t=\alpha+\beta I t+u t
$$

A hipótese nula é $\beta=1$ e $u_{t}$ estacionário. Em outras palavras, se a poupança e o investimento são não estacionários, a hipótese nula é $\beta=1$ e que poupança e investimento são cointegrados com vetor de cointegração $(1,-1)^{\prime}$ '. Um teste relacionado é o teste da estacionariedade do déficit em conta corrente. Este teste é relacionado ao teste de $\beta=1$ porque o déficit em conta corrente restringe os parâmetros da regressão de cointegração a $\alpha=0$ e $\beta=1$.

Mas estes testes, obviamente, são idênticos à releitura da equação original de Feldstein e Horioka (1980) feita pela abordagem de raiz unitária e cointegração. Isto porque a equação de regressão original, estimada com as variáveis em níveis, simplesmente ignora a dinâmica intertemporal enfatizada pelos modelos teóricos e consiste, portanto, numa relação de longo prazo. No longo prazo, como discutido anteriormente, a poupança e o investimento estão amarrados pela restrição orçamentária intertemporal e, somente no curto prazo, choques podem fazer com que a poupança e o investimento divirjam dos seus níveis de "steady-state”. Além disso, dado que a regressão de "cross-section" mede o coeficiente médio de longo prazo, o que ela está na verdade captando é o coeficiente dado pela restrição de solvência e não o grau de mobilidade de capitais.

\section{CONCLUSÕES}

Feldstein e Horioka (1980) usaram a correlação investimento-poupança para avaliar o grau de mobilidade de capitais. $\mathrm{Na}$ amostra original de dados que eles examinaram, regressões de "cross-section" do investimento na poupança resultaram em coeficientes próximos da unidade, indicando que nos países da OCDE o grau de mobilidade de capitais era baixo. Esse mesmo resultado foi obtido para outras amostras e para diferentes períodos de tempo, o que fez o coeficiente de "crosssection" próximo de 1 ser considerado um "puzzle”.

\footnotetext{
${ }^{6}$ A equivalência entre cointegração e o atendimento da restrição de valor presente foi demonstrada, por exemplo, em Hakkio e Rush (1991) num contexto determinista e em Ahmed e Rogers (1995) num contexto estocástico.
} 
O objetivo desse artigo é argumentar que não existe "puzzle" algum e que o coeficiente próximo da unidade pode ser melhor compreendido quando se usa uma fundamentação teórica adequada para interpretar a regressão original de Feldstein e Horioka. Caso sejam utilizados modelos intertemporais de economias abertas e a literatura empírica que usa esses modelos para testar sustentabilidade, é possível demonstrar que a condição de solvência requer que a conta corrente seja estacionária uma vez que não é possível a qualquer país incorrer indefinidamente num jogo Ponzi com sua dívida. A estacionariedade da conta corrente implica, ainda, que poupança e investimento cointegram com coeficiente unitário uma vez que, por definição, a conta corrente iguala a poupança menos o investimento. Dado que a regressão de "cross-section" mede o coeficiente médio de longo prazo ela está, então, captando o coeficiente unitário implicado pela condição de sustentabilidade/ solvência e não o grau de mobilidade de capitais.

\section{REFERÊNCIAS BIBLIOGRÁFICAS}

AHMED, S. and ROGERS, J.H. (1995) “Government Budget Deficits and Trade Deficits: are Present Value Constraints Satisfied in Long-term Data?”. Journal of Monetary Economics, 36: 351-74.

ARGIMÓN, I. and ROLDÁN, J.M. (1994) "Saving, Investment and International Capital Mobility in EC Countries”. European Economic Review, January, 38: 59-67.

BAGNAI, A. and MANZOCCHI, S. (1996) "Unit Root Tests of Capital Mobility in the Less Developed Countries”. Weltwirtschaftliches Archiv, vol.132 (3): 545-557.

BAYOUMI, T. (1990) "Saving-investment Correlations: Immobile Capital, Government Policy, or Endogenous Behavior”. IMF Staff Papers, June, 37:360-387.

COAKLEY, J., KULASI, F., and SMITH, R. (1996) "Current Account Solvency and the Feldstein-Horioka Puzzle”. Economic Journal, 106: 620-627.

CORSETTI, G., PESENTI, P. and ROUBINI, N. (1998a) What Caused the Asian Currency and Financial Crisis? Part I: Macroeconomic overview

CORSETTI, G., PESENTI, P. and ROUBINI, N. (1998b) What Caused THE Asian Currency and Financial Crisis? Part II: The policy debate

DOOLEY, M., FRANKEL, J. and MATHIESON, D. (1987) "International Capital Mobility: What do Saving-Investment Correlations Tell Us?”. IMF Staff Papers, 34(3): 503-530.

FELDSTEIN, M. (1983) "Domestic Saving and International Capital Movements in the Long Run and in the Short Run”. European Economic Review, 129-151.

FELDSTEIN, M. and BACCHETTA, P. (1991) "National Saving and International Investment". In National Saving and Economic Performance, edited by D. Bernheim and J. Shoven. Chicago: University of Chicago Press, 201-220.

FELDSTEIN, M. and HORIOKA, C. (1980) "Domestic Saving and International Capital Flows". Economic Journal, June, 90:314-329.

GHOSH, A.R. and OSTRY, J.D. (1995) “The Current Account in Developing Countries: A Perspective from the Consumption-Smoothing Approach", The World Bank Economic Review, vol. 9 (2): 305333.

GUNDLACH, E. and SINN, E. (1992) “Unit Root Tests of the Current Account Balance: Implications for International Capital Mobility”. Applied Economics, June, 24: 617-625.

HAKKIO, C.S. and RUSH, M. (1991) “Is the Budget Deficit “Too Large?”. Economic Inquiry, 429-445. HAMILTON, J. and FLAVIN, M. (1986) "On the Limitations of Government Borrowing: a Framework for Empirical Testing”. American Economic Review, 76: 353-373. 
HUSTED, S. (1992) “The Emerging U.S. Current Account Deficit in the 1980s: a Cointegration Analysis". The Review of Economics and Statistics, 159-66.

ISSLER, J.V. and LIMA, L.R. (2000) "Public Debt Sustainability and Endogenous Seigniorage in Brazil: Time Series Evidence from 1947-1992”. Journal of Development Economics, v.62 (13).

LUPORINI, V. (2000) "Sustainability of the Brazilian Fiscal Policy and Central Bank Independence". Revista Brasileira de Economia, v. 54 (2): 201-226.

MILLESI-FERRETTI, G.M. and RAZIN, A. (1996a) "Sustainability of Persistent Current Account Deficits", NBER Working Paper, n. 5467.

MILLESI-FERRETTI, G.M. and RAZIN, A. (1996b) "Current Account Sustainability: Selected East Asian and Latin American Experiences”, NBER Working Paper, n. 5791.

MURPHY, R.G. (1984) "Capital Mobility and the Relationship Between Saving and Investment in OECD Countries”. Journal of International Money and Finance, vol.3: 327-342

OBSTFELD, M. and ROGOFF, R. (2000) “The Six Major Puzzles in International Macroeconomics: is There a Common Cause?”, NBER Working Paper 7777.

OBSTFELD, M. (1986) "Capital Mobility in the World Economy: Theory and Measurement", CarnegieRochester Conference Series on Public Policy, Spring, 1-24.

PASTORE, A.C. (1995) "Déficit Público e a Sustentabilidade do Crescimento das Dívidas Interna e Externa, Senhoriagem e Inflação : uma Análise do Regime Monetário Brasileiro”, Revista Brasileira de Econometria, v. 14 (2):177-234.

PONTA, A.F. (1996) "A Sustentabilidade do Endividamento Externo no Brasil: uma Análise de Cointegração". Pesquisa e Planejamento Econômico, v. 23 (3): 325-416.

ROCHA, F. (1997) "Long Run Limits on the Brazilian Government Debt". Revista Brasileira de Economia, v. 51 (4): 447-470.

ROCHA, F. e BENDER, S. (2000) "Present Value Tests of the Brazilian Current Account". Economia Aplicada, v. 4 (2): 203-222.

SAWADA, Y. (1994) "Are Heavily Indebted Countries Solvent? Tests of Intertemporal Borrowing Constraints". Journal of Development Economics, 45: 325-37.

SINN, S. (1992) "Saving-Investment Correlations and Capital Mobility: On the Evidence from Annual Data”. Economic Journal, September, 102: 1162-1170.

TESAR, L. (1991) “Saving, Investment, and International Capital Flows”. Journal of International Economics, 31: 55-78.

TREHAN, B. and WALSH, C.E. (1991) "Testing Intertemporal Budget Constraints: Theory and Applications to US Federal Budget and Current Account Deficits". Journal of Money, Credit, and Banking, v. 23: 203-223.

WONG, D. (1990) “What Do Saving-Investment Relationships Tell Us About Capital Mobility?”, Journal of International Money and Finance, vol. 9: 60-74. 